\title{
Development automated capture device for picking apples
}

\author{
Dmitriy Khort, Alexey Kutyrev*, Igor Smirnov, and Daniil Pupin \\ Federal Scientific Agroengineering Center VIM, 1-st Institutsky proezd, 5, Moscow, 109428, Russia
}

\begin{abstract}
In the system of the mechanized process of cultivation of fruit crops, harvesting is an important final stage, which requires the development of new, convenient, including those that do not damage the fruit, automated technical devices. As a result of a series of experiments, the operability of the developed automated fruit picking device was confirmed, it gently captures the fruit and reliably holds it. The operating modes of the automated device for picking fruits have been established. The following characteristics of the device for picking fruits were revealed: the time for a complete capture of the apple is from 1.5 seconds to 2 seconds, depending on their size, the maximum size of the captured apple is $85 \times 80 \mathrm{~mm}$, the maximum weight of the captured apple is $400 \mathrm{~g}$. The permissible pressure of the paws of the device on the fruit is determined ... It was found that with the force of the linear stepper motor on the pusher in $8 \mathrm{~N}$, the safety of the apple is ensured with minimal damage, while the apple is securely fixed in the capture paws.
\end{abstract}

\section{Introduction}

In the system of the mechanized process of cultivation of fruit crops, harvesting is an important final stage, which requires the development of new, convenient, including nonfruit-damaging automated technical devices installed on robotic platforms capable of autonomously harvesting fruits, therefore, developments for the creation of automated devices for removing fruits of fruit plantations with minimal damage (or without them) at a height of up to 5 meters are an urgent task [1,2]. The existing models of industrial robots cannot be directly applied to perform technological processes of loading, unloading, sorting and harvesting apples [3,4]. In particular, for the latter, it is necessary to develop special actuators, capture devices and new algorithms for their control for harvesting fruit plantations in the field $[5,6]$. To establish the optimal design parameters of the removal devices, substantiate the parameters of their control system and the successful introduction of this technology into the production process, it is necessary to conduct scientific research. A self-propelled robotic technical device with a developed automated capture-manipulator will make it possible to carry out a high-quality technological operation of harvesting fruits in industrial garden plantings without human intervention.

\footnotetext{
${ }^{*}$ Corresponding author: alexeykutyrev@gmail.com
} 


\section{Materials and Methods}

Analysis of existing devices for picking fruits showed that the optimal type of device is a concentric three-fingered grip. It combines the features of a rotary and a sliding capture, providing significantly greater reliability of holding the fetus than other types of capture at the same force values. The center of this grip does not move from its original position when opening and squeezing its fingers, thus, a linear actuator can be used as an actuator for automating the fruit collection process.As a result of the research carried out in the CAD software environment Autodesk Fusion 360, a 3D model of the fruit picker was developed (Fig.1).

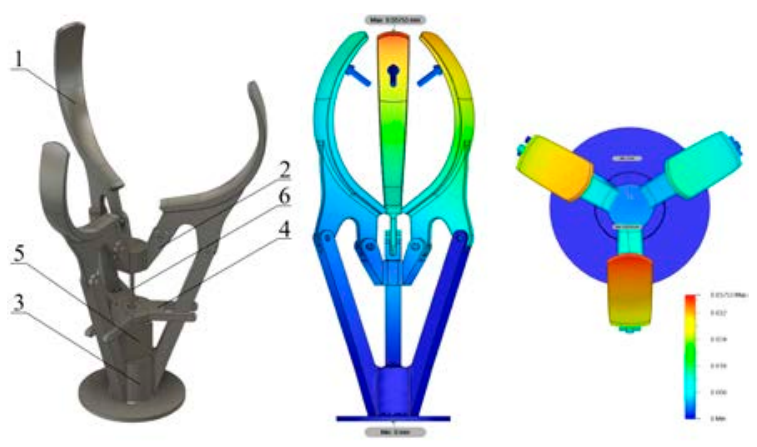

Fig. 1. 3D model of the device for picking fruits, the results of the analysis of the stress-strain state of the structure: 1 - capture paws; 2 - pusher; 3 - base; 4 - stepper motor mount; 5 - linear stepper motor; 6 - rod of a linear stepper motorFig. 1. 3D model for the removal of the fruit, the results of the analysis stress-strain state of the structure: 1 - grip paws; 2 - pusher; 3 - base; 4 - fastening of stepper motor; 5 - linear stepper motor; 6 - rod linear stepper motor

A block diagram of the automated control system for fetal removal has been de-veloped, which includes a set of hardware and software tools, a microcontroller (MC), a fetal tear sensor (DSP), a fetal pressure sensor (DDP), a stepper motor driver (SMD), a linear actuator (LA), and a computer for manipulating a manipu-lator with a fruit recognition system (K) (Fig.2).

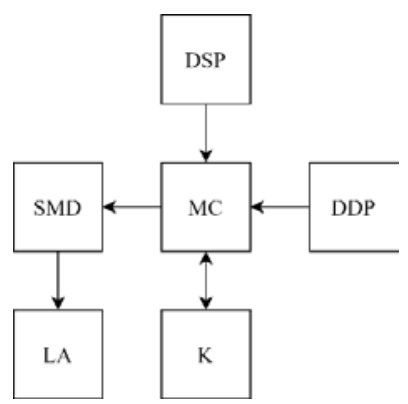

Fig. 2. Structure of the control system for automated fruit removal

All hardware elements are located inside the base of the fruit pick-up device; data from pressure sensors and fetal stripping come through the analog ports. Communication with the computer is carried out using the standard UART serial interface. The software part of the device is a software code that controls the position of a stepping linear actuator, processes data from pressure and fetal failure sensors, reads and sends data to a computer. An Atmel ATmega328p in a 28-PDIP package is used as a programmable microcontroller (Fig. 3). The used eight-bit microcontroller has good performance, and due to the $60 \mathrm{~nm}$ process technology, it has low power consumption. Depending on the applied voltage in the range from $1.8 \mathrm{~V}$ to $5.5 \mathrm{~V}$, this microcontroller can operate at a frequency of $4 \mathrm{MHz}$ to 20 
MHz. The internal clock source of the Atmega328p operates at $8 \mathrm{MHz}$, but since there are devices in the capture control circuit that need to be processed at high speed, an external clock source is used at $16-20 \mathrm{MHz}$.

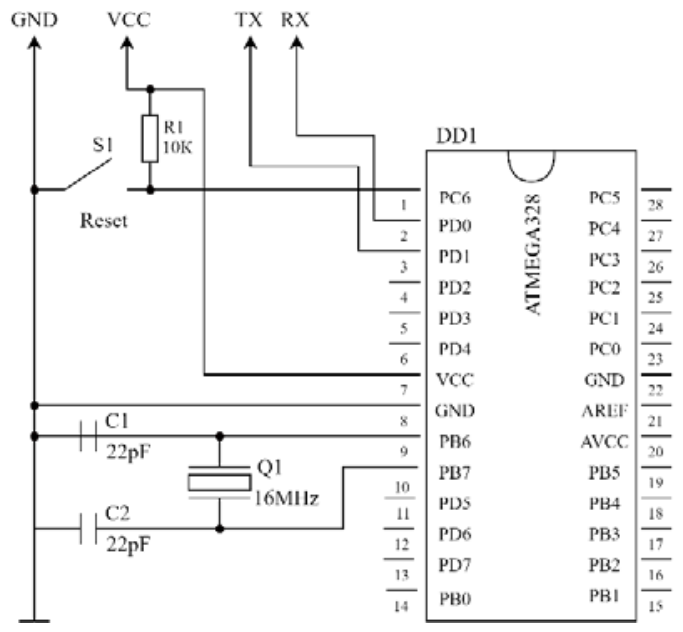

Fig. 3. Atmega328p microcontroller connection diagram

To increase the clock frequency of the microcontroller, a crystal oscillator is connected, an external clock source is $16 \mathrm{MHz}$; to reboot the microcontroller, a button S1 is provided, which closes contact 1 with GND. The RX and TX pins are for connecting the programmer. The USB programmer USBASPV is used as a programmer.Development of a fetal abruption sensor. When performing a technological operation of collecting fruits in the field, a robotic arm must pick the fruit, and then move it to a container for storage. If the grip cannot accurately record the moment of the fruit's break, then it will continue to pull the fruit after it has been lifted off the stalk. The presence of a fetal abruption sensor in the system will reduce the time for collecting fruits, since there will be no unnecessary movements of the manipulator. When the fruit is torn off by grip, if you do not hold the branch on which the fruit is hanging, the stalk will not separate from the branch immediately; some effort must be used to break it. At the same time, at the moment when the stalk is detached from the branch, the capture receives a sharp acceleration towards the separation of the fruit. This sharp acceleration will show the moment of separation of the fruit from the branch. To register this acceleration, an accelerometer sensor is used. When choosing an accelerometer, it is necessary to take into account accelerations in all directions; for this, a three-axis accelerometer ST Microelectronics LIS331DLHTR is used, supply voltage 1.5-3.6 V, interface type i2c, spi, sensitivity $0.1 \mathrm{~m} / \mathrm{s} 2$ connected according to the diagram (Fig. 4).

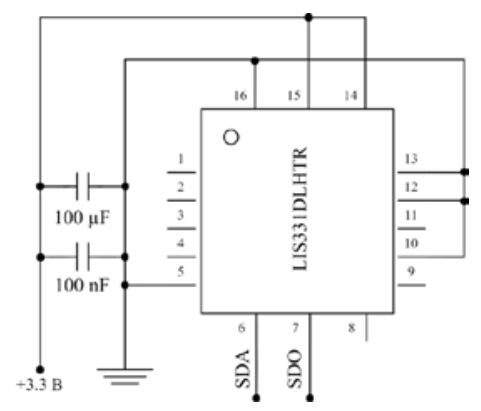

Fig. 4. Wiring diagram for the ST Microelectronics LIS331DLHTR accelerometer sensor 
The ST Microelectronics LIS331DLHTR accelerometer is connected to an ATmega 328 microcontroller via a spi serial peripheral interface. To power the accelerometer, an Analog Devices ADP3338AKCZ-3.3RL7 voltage regulator with a low voltage drop and voltage stabilization of $3.3 \mathrm{~V}$ is used, connected according to the diagram (Fig. 5).

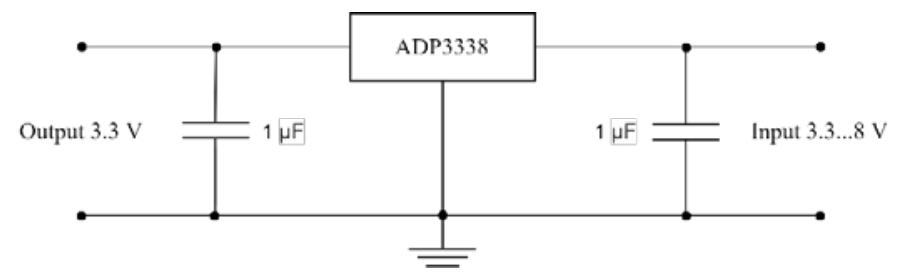

Fig. 5. Wiring diagram for voltage regulator Analog Devices ADP3338

To calibrate the fetal abruption sensor, it was attached to a prototype of an automated fruit picker, which worked in conjunction with a manipulator. The manipulator was operated manually using a remote control. The robotic arm issued a work to collect one apple, it began from the moment the breakdown began, and ended with the moment the apple was folded into a container for transportation. The fetal abruption sensor during the manipulator operation constantly changes its position, thereby the measurement on one coordinate axis will be unreliable, for accurate registration of the moment of fetal abruption, the acceleration of the capture device was measured along three coordinates $\mathrm{x}, \mathrm{y}$, $\mathrm{z}$, the location of the coordinates on the accelerometer microcircuit is shown on Figure 6.

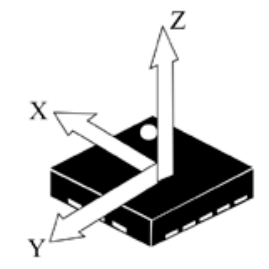

Fig. 6. location of coordinates on the accelerometer sensor

A computer was connected to the fetal abruption sensor, which continuously took readings from the accelerometer sensor. As a result, we obtained acceleration data along the x-axis (Fig. 7), along the y-axis (Fig. 8), and along the z-axis (Fig. 9).

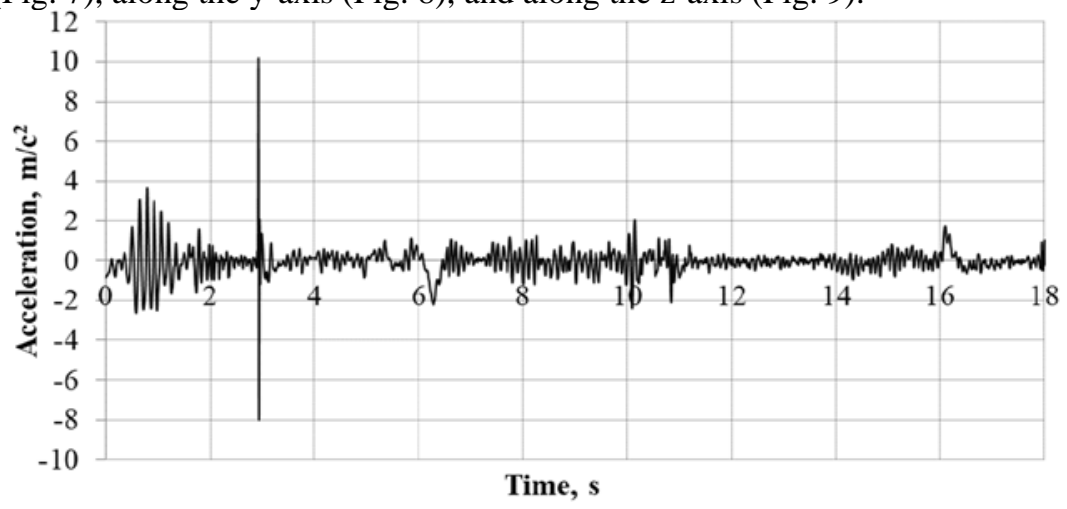

Fig. 7. Acceleration along the $x$-axis 


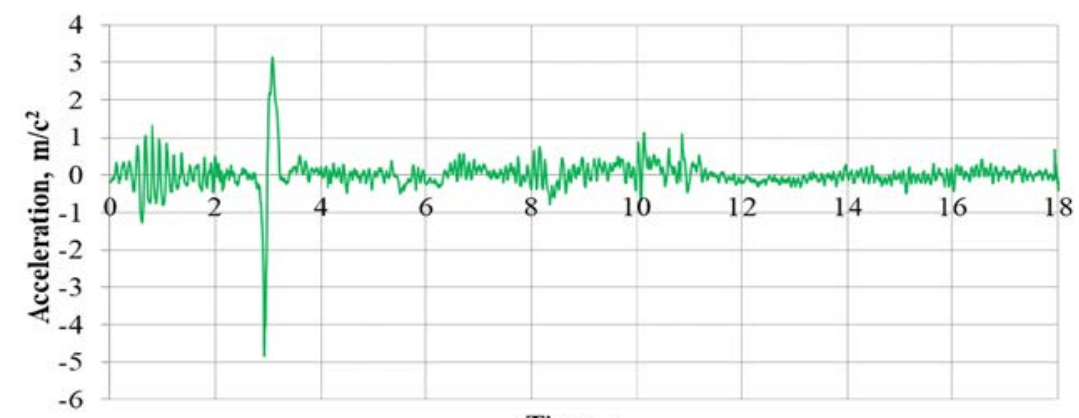

Time, s

Fig. 8. Acceleration along the y-axis

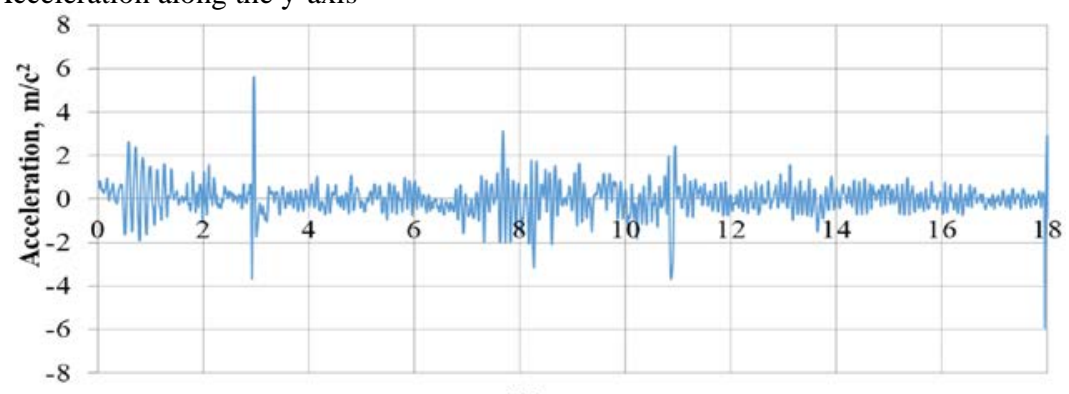

Time, s

Fig. 9. The acceleration along the z-axis

In the interval from 0 seconds to 2,7 seconds, vibrations with a maximum amplitude along the $\mathrm{x}$-axis of $3,8 \mathrm{~m} / \mathrm{s}^{2}$ are visible along all three axes, which are performed by the device for picking fruits with a fixed apple in the capture paws, at the moment the manipulator begins to move. From 2.8 seconds, there is a sharp increase in acceleration, this is the moment of separation of the stalk from the tree branch. After detachment of the stalk, the manipulator picks up acceleration with a maximum amplitude along the $\mathrm{x}$-axis of $10 \mathrm{~m} / \mathrm{s}^{2}$. In the interval from 3,2 seconds to 18 seconds, the manipulator moves the apple into the container for its transportation. An acceleration module was found to process the received data.

On the graph of the acceleration modulus values (Fig. 10), in the interval from 2,8 seconds to 3,2 seconds, the acceleration modulus increases with a maximum value of up to $11,8 \mathrm{~m} / \mathrm{s}^{2}$.

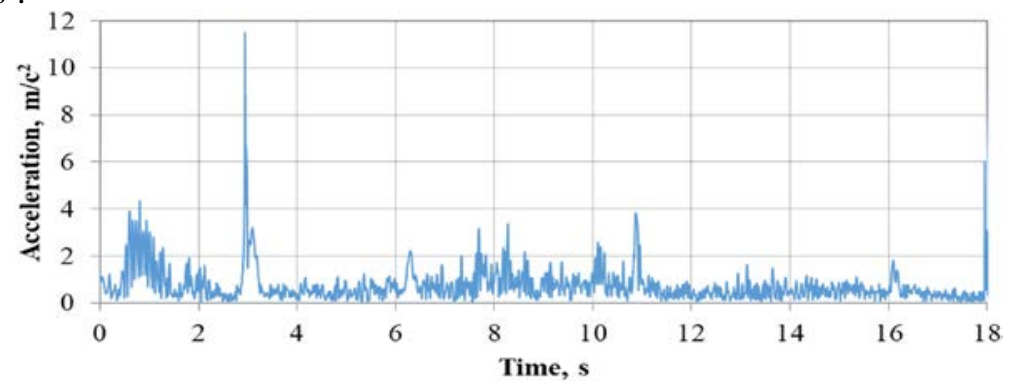

Fig. 10. The acceleration along the z-axis

When reading the signal from the accelerometer, the microcontroller determines the maximum value of the acceleration module, this value will be the moment when the stalk is detached from the tree branch. Selecting and connecting a stepper motor driver. When choosing a stepper motor driver, Table 1 , it is necessary to take into account the following 
parameters: driver dimensions, type of connection to the microcontroller (STEP / DIR protocol), voltage per motor winding from $9 \mathrm{~V}$, current per winding from $1 \mathrm{~A}$, type of winding connection bipolar. In addition to the dimensional and technological parameters of the stepper motor driver, the weight of the driver must be taken into account. Drivers with a massive heatsink or drivers with external elements are excluded, and drivers with external power switches are also excluded. As a result of the research, the A4988 driver was selected, operating in the required range of electrical parameters: supply voltage: 8-35 V, micro-step mode $1,1 / 2,1 / 4,1 / 8,1 / 16$, logic voltage: 3-5,5 V, overheating protection, maximum current per phase: 2,5 A, size: $20 \times 15 \mathrm{~mm}$. The stepper motor driver A4988 is connected according to the diagram (Fig. 11).

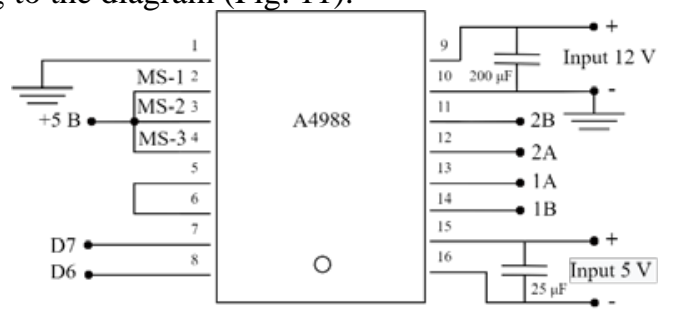

Fig. 11. Wiring diagram motor driver A4988

The A4988 stepper motor driver, when commanded to stop the motor, applies current to its two windings at the same time; in the case of a linear stepper motor, this action is not required to stop it. The linear stepper motor operates above the rated values, therefore, in braking mode, a large current is applied to the motor, which leads to it heating up to $80^{\circ} \mathrm{C}$. In order to prevent overheating of the motor, a power control circuit for the stepper motor driver has been developed (Fig. 12).

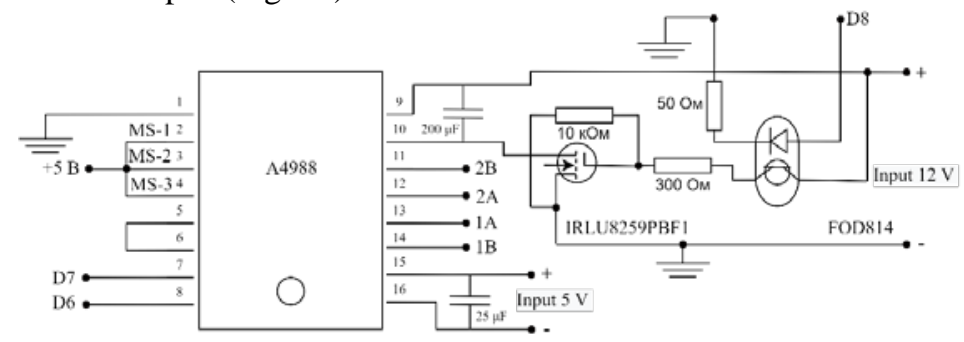

Fig. 12. The power management scheme A4988

When the stepper motor is turned on, the microcontroller sends a signal to the FOD814 optocoupler, which opens the IRLU8259PBF1 transistor and through it supplies power to the stepper motor driver. Development of a fetal pressure sensor. When capture the apple with the paws of the robotic capture, it is necessary to provide such an effort at which the apple will not be damaged, but at the same time it will be securely fixed in the paws. To automate the regulation of the degree of pressure of the capture paws on the fetus, an algorithm has been developed that automatically selects the degree of pressure of the capture paws on the fetus. To understand the degree of pressure of the capture paws on the fetus, it is necessary to develop a fetal pressure sensor. The fetal pressure sensor works on the principle of changing the current in the winding of a stepper motor, the greater the current in the winding, the greater the pressure the capture paws grasp the fetus. It is necessary to measure the current in the winding using a current sensor, the current data is processed by a microcontroller. A fetal pressure sensor consists of a current sensor connected to a microcontroller. The current sensor is connected to one of the stepper motor windings (Fig. 13). 


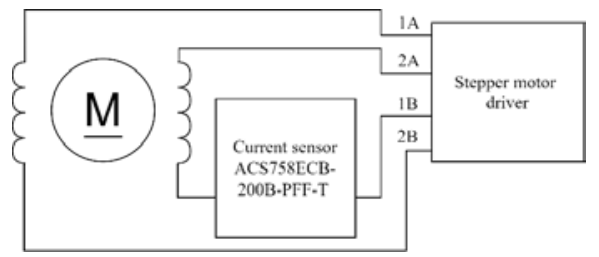

Fig. 13. Wiring diagram of the current sensor to the stepper motor driver

Allegro MicroSystems ACS758ECB-200B-PFF-T was used as a current sensor, this sensor is capable of measuring current up to 200 A with an accuracy of $3 \mathrm{~mA}$. The sensor is connected to an open circuit of the measured circuit. The current reading is converted to voltage, which should be converted to the measured current value.

\section{Results and Discussion}

As a result of the research carried out, an intelligent control system for the equipment of a robotic device for picking fruits has been developed, which is able to determine in the shortest time the moment when the fetus is ripped off the stalk, and is also able to control the degree of pressure of the capture paws on the fetus. 3D printing makes the capture. The material used is polyamide 12 (PA 12), better known as nylon, this material is characterized by high strength, wear resistance, resistance to weathering. After assembling a prototype of an automated device, laboratory experiments were carried out. The functionality of the developed capture for harvesting fruits, namely apples, has been tested. To do this, the capture was installed on a manipulator, after which a series of experiments was carried out on an artificial model of an apple tree with fruits of various size parameters (Fig. 14.).

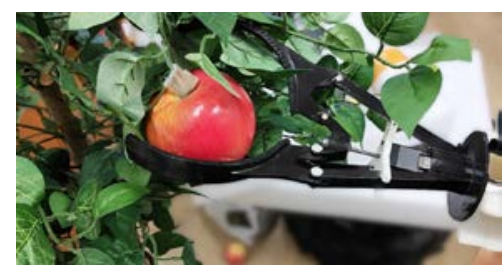

Fig. 14. Process of conducting a laboratory experiment, capturing the fetus with a developed device

As a result of a series of experiments, the performance of the grip was confirmed; it gently grips the fetus and reliably holds it. The following characteristics of the grip were revealed: the time to grip the apple is from 1.5 seconds to 2 seconds, depending on their size, the maximum size of the gripped apple is $85 \times 80 \mathrm{~mm}$, the maximum weight of the captured apple is $400 \mathrm{~g}$. The permissible pressure of the capture paws on the fruit is determined. The grabbing of apples was carried out with various efforts, after which the test samples were photographed and from the photograph, using the written code with the OpenCV library, the place of damage was recognized and their area was determined (Fig. 15).

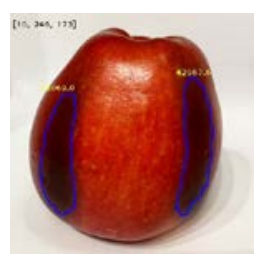

Fig. 15. Analysis of the degree of damage to an apple after a laboratory experiment 
The area of apples for a threefold repetition of the experiment is determined in pixels (elements of a two-dimensional digital image). The found area in pixels, knowing the focal length of the camera used (Basler acA1920-155uc, Sony IMX174 CMOS sensor), is converted into millimeters. Having determined the area of influence of the grip on the apple, $\mathrm{S}=0.001421 \mathrm{~m}^{2}$, the limiting values of pressure on the fruit surface were determined (Table 1).

Table 1. Results of the analysis of the obtained data of the field experiment

\begin{tabular}{|c|c|c|c|c|c|c|}
\hline Parameter & \multicolumn{7}{|c|}{ Value } \\
\hline Force of action, H & 12 & 10 & 8 & 6 & 4 & 2 \\
\hline Pressure, Pa & 13780,1 & 6891,7 & 5513,4 & 3445,8 & 2415,4 & 1515,7 \\
\hline $\begin{array}{c}\text { Average damage } \\
\text { percentage, \% }\end{array}$ & 7,79 & 3,56 & 0,72 & 0,57 & 0,25 & 0,14 \\
\hline
\end{tabular}

The efforts were measured using the developed fetal pressure sensor by measuring the current strength, which is in direct proportion to the applied force. It was found that with the force of the linear stepper motor on the pusher in $8 \mathrm{~N}$, the safety of the apple is ensured with minimal damage, while the apple is securely fixed in the capture paws.

\section{Conclusions}

As a result of the research carried out, an experimental model of an automated device for picking fruits and a system for its automated control have been developed. The conducted laboratory experiments made it possible to determine the operating modes of the automated device for picking up fruits and to confirm its performance. The use of automated devices with computer vision systems in agricultural units represents a huge potential for automation of accounting and decision control. To establish optimal operating conditions and the successful introduction of this technology into the production process, additional research is needed to fully optimize the technology for a wide range of conditions existing in agricultural production of horticultural crops. The results obtained will become the basis for the transition of agricultural sectors to a new technological level of production.

\section{References}

1. D. Khort, A. Kutyrev, R. Filippov, N. Kiktev and D. Komarchuk, Robotized Platform for Picking of Strawberry Berries, Science and Technology (PIC S\&T), pp. 869-872 (2019)

2. D. Khort, A. Kutyrev, I. Smirnov, V. Osypenko, N. Kiktev Computer Vision System for Recognizing the Coordinates Location and Ripeness of Strawberries, Data Stream Mining \& Processing, Communications in Computer and Information Science, 1158, Springer, Cham (2020)

3. M.M. Sofu, O. Er, M.C. Kayacan, B. Cetisli. Design of an automatic apple sorting system using machine vision, Computers and Electronics in Agriculture, 127, pp 395-405 (2016)

4. B. Zhang, W. Huang, L. Gong, J. Li, C. Zhao, C. Liu, D. Huang Computer vision detection of defective apples using automatic lightness correction and weighted RVM classifier, Journal of Food Engineering, 146, pp 143-151 (2015)

5. A.K. Bhatt, D. Pant. Automatic apple grading model development based on back propagation neural network and machine vision, and its performance evaluation, AI \& Soc, 30, pp 45-56 (2015)

6. O. Kleynen, V. Leemans, M.-F. Destain Development of a multi-spectral vision system for the detection of defects on apples, Journal of Food Engineering, 69, pp 41-49 (2005) 